\title{
The controversy regarding hand-assisted colorectal resection
}

\author{
Sharon Stein · Richard L. Whelan
}

Received: 10 August 2007/Accepted: 16 October 2007/Published online: 1 November 2007

(C) Springer Science+Business Media, LLC 2007

The technique of hand-assisted colectomy was introduced in the 1990s but initially was not widely embraced by the minimally invasive community $[1,2]$. However, in the past 4-6 years the popularity of hand-assisted methods has increased considerably. One reason for the surge in interest was the publication of the Clinical Outcomes of Surgical Therapy (COST) findings, which suggested that laparoscopic colectomy for cancer was associated with comparable oncological outcome and improved short-term results [3]. Many surgeons who had not been performing laparoscopic colectomy now felt it was safe to do so and, thus, began utilizing minimally invasive methods. Another important reason for the increased utilization of handassisted methods in recent years was the introduction of second- and third-generation devices that are far easier to use and are more versatile than the initial devices.

As the use of hand-assisted techniques has increased, a rift has developed in the minimally invasive world regarding the appropriateness and need for hand-assisted methods. Strong proponents believe that hand-assisted methods are appropriate for all left-sided colorectal resections whereas others feel strongly that, for an experienced minimally invasive surgeon, there is no need for hand-assisted methods at all. Until recently, the relatively sparse published literature on the subject has been insufficient to settle the dispute. However, the recent multicenter randomized study presented at the 2007 American Society

S. Stein

New York Presbyterian Hospital, New York, NY, United States

R. L. Whelan $(\bowtie)$

College of Physicians and Surgeons, Columbia University,

New York, NY, United States

e-mail: rlw3@columbia.edu of Colon and Rectal Surgeons (ASCRS) meeting sheds further light on the subject. This study suggested that handassisted colectomy is associated with time savings of 33 and $57 \mathrm{~min}$ for left segmental colectomy and total abdominal colectomy, respectively. Importantly, the shortterm clinical outcomes of hand-assisted procedures were comparable to those obtained using straight laparoscopic methods [4]. The mean incision length for the hand-assisted group was $8.2 \mathrm{~cm}$ as opposed to $6.1 \mathrm{~cm}$ for the laparoscopic group. Despite these findings, the controversy continues.

Laparoscopic "purists" believe that there is little or no place for hand-assisted methods because the straight laparoscopic approach results in a smaller final incision. Since the whole point of laparoscopy is to minimize abdominal wall trauma, they can find no acceptable rationale for "giving back" incision length to accommodate hand methods. Some feel that the abdominal wall trauma incurred with hand-assisted operations more resembles an open rather than a minimally invasive operation. They maintain that, with the proper skills and training, straight laparoscopic resections are feasible and clearly preferable to hand-assisted approaches. Straight laparoscopic proponents also worry that surgeons who exclusively learn handassisted methods will never master the two-handed technique and are not likely to cross the bridge to straight laparoscopic resection. Thus, a cadre of hand-assisted surgeons is likely to arise who will be doing a second best type of resection.

Hand-assisted proponents believe that, clinically, for the majority, the recovery and morbidity of hand-assisted patients is very close to if not the same as patients that undergo straight laparoscopic resection. Further, they point out that the difference in overall incision size between the two approaches is not that great. Supporters also believe 
that hand techniques allow minimally invasive operations in a subset of patients, namely the very obese and large, in whom a successful straight laparoscopic resection is not likely. Advocates further point out that, in a sizable percentage of patients in whom a standard laparoscopic resection is feasible, the patient's body mass index (BMI), body habitus, or the overall size and bulk of the specimen are such that an extraction incision will be required that is close to, if not the same size as, the incision necessary for a straight laparoscopic resection. Supporters also believe that hand-assisted methods are the logical next step in straight laparoscopic cases that require conversion; they point out that the majority of such cases can be completed via the hand-assisted approach and a larger laparotomy can be avoided. Lastly, proponents point out that it is easier and more practical to teach hand techniques rather than pure laparoscopic methods to already trained surgeons. The same is true for general surgery residents who have limited time on the colorectal service. The "hard core" enthusiasts utilize hand-assisted methods for all left-sided and rectal resections and see few indications for straight laparoscopic colorectal resections on the left.

The "hard line" positions for both straight laparoscopic and hand methods have weaknesses and are not tenable. As is often the case, a middle of the road position is more logical and easier to defend. Hand-assisted techniques are intrinsically different from pure laparoscopic methods. They are invaluable for a proportion of patients in whom a fully laparoscopic operation is not feasible or would otherwise be exceedingly difficult; thus, they increase the overall percentage of patients to whom a minimally invasive operation can be offered. Hand-assisted methods are also valuable because they are associated with a time savings. However, hand-assisted methods are not for all patients; thin and low-BMI patients are most often better off with a straight laparoscopic operation.

The available clinical results suggest that it is logical to offer minimally invasive operations to as many patients as possible. Is a straight laparoscopic resection feasible in all patients? High-BMI and very tall and broad patients are problematic laparoscopically for numerous reasons including difficult port placement, limited exposure, retraction difficulties, bulky omentum, and obscure anatomy and tissue planes. Because of these challenges, some laparoscopic surgeons have BMI cutoffs, above which open methods are universally employed. Hand-assisted methods facilitate surgery in obese patients because tactile sensation is restored and it is easier to retract, expose, and find dissection planes. The ability to palpate and, at times, to dissect blindly with one or several fingers, once the proper plane has been established, is an especially important benefit of hand-assisted methods in this population.
Not infrequently, even with hand-assisted methods, in the very obese the goals of the surgeon need to be scaled back in regards to the proportion of the case to be completed laparoscopically, since in a considerable percentage it is not possible to fully complete the resection under pneumoperitoneum. Certain parts of the procedure may need to be carried out via open means via an enlarged hand incision (the hybrid laparoscopic/open approach). Nevertheless, provided certain key elements of the operation can be completed via minimally invasive means, it is usually possible to limit the final incision to the 9 to $12 \mathrm{~cm}$ range, which is usually less than half the length of incision needed if open methods were employed [5]. In the case of leftsided or low anterior resections, if the splenic flexure can be fully mobilized then the patient will benefit. In the case of right colectomy, as long as the colon can be fully mobilized the rest of the case can be done via the hand port incision or a slightly extended incision. It is important to remember that open surgery in the very obese is also difficult and most often requires a larger than usual incision. Although minimally invasive operations in the very obese are a challenge, in no other group are the benefits as great. It is the opinion of the authors, based on many cases, that hand-assisted methods allow successful resection via a hand-assisted hybrid approach in the subset of very obese patients in whom a successful straight laparoscopic resection would not be feasible.

Another challenging group of patients are those with bulky pathology or complex anatomy such as colovesical fistula, dense adhesions involving adjacent bowel loops or other organs (post RT, inflammatory, or postsurgical), or locally invasive tumors. In these patients it may prove impossible to safely mobilize and resect the bowel laparoscopically. In particular, in patients with large or bulky rectal tumors it may prove very difficult to safely transect the distal rectum below the cancer laparoscopically. Conversion rates are higher in these difficult patients. In addition, even if the bowel is fully mobilized and resected laparoscopically, a larger incision than usual is often required to accommodate extraction of bulky specimens. It makes sense to use hand-assisted methods in this setting since the hand inside will facilitate the dissection, and help clarify the anatomy. Also, since a larger than usual incision is often needed to remove the specimen regardless, why not make full use of the incision from the outset?

In patients undergoing total abdominal colectomy or proctocolectomy a strong case can be made for handassisted methods. If straight laparoscopic methods are used these cases are usually very long. In the Marcello et al. study presented at the 2007 ASCRS meeting, the use of hand-assisted methods in this setting was associated with a time savings of 56 min for the total abdominal colectomy portion of the operation. Further, in total proctocolectomy 
cases where an ileal pouch is constructed and a pouch anal or pouch rectal anastomosis performed, a decent sized incision is needed regardless. Hand-assisted methods are also logical for a substantial percentage of low anterior resections, regardless of tumor bulk, because of the difficulty encountered when trying to transect the distal rectum cleanly, especially in a narrow male pelvis.

Putting aside the challenging patient subgroups discussed above, in the majority of patients coming to resection in the US with a middle range BMI and moderately sized pathology a straight laparoscopic approach is feasible with an acceptable conversion rate and a mean incision length of $5-7 \mathrm{~cm}$. Is there a way to justify the use of hand methods in this population? Since the average incision required for a hand-assisted resection is between 8 and $9 \mathrm{~cm}$, the difference, in regards to abdominal wall trauma, is $1-4 \mathrm{~cm}$. The results of the recently published randomized trial, mentioned above, suggest that, despite the fact that the mean laparoscopic incision was significantly shorter $(6.1$ versus $8.2 \mathrm{~cm})$ that there was no significant difference in the short-term outcome [4]. Thus, although the straight laparoscopic approach is associated with a significantly smaller incision in these patients, the difference is not that great and, as per the results of the Marcello et al. study, does not appear to be associated with a prolonged or more morbid short-term recovery. Of note, a well-performed single-center prospective Australian study comparing straight laparoscopic and hand-assisted low anterior resections reported a significantly longer time until flatus (3.4 versus 1.8 days) and a greater mean duration of narcotic usage (3.0 versus 1.5 days) in the hand-assisted group. Despite these differences the overall length of stay was similar (5.9 versus 5.8 days). This well-performed study suggests there are some differences in recovery in favor of straight laparoscopic methods [6].

When the straight laparoscopic surgeon who is not familiar with hand-assisted methods needs to convert, he performs a laparotomy large enough to allow completion of the case via open methods. The available data suggests that a large proportion of these cases can be completed via the hand-assisted method. In the recently presented randomized Hand-Assisted Laparoscopic (HAL) versus open left and total abdominal colectomy trial, five of the six conversions in the straight laparoscopic group were to a handassisted approach, which permitted safe completion of the case via the hand device incision [4]. The hand device allows the case to be continued via minimally invasive means; the intracorporeal hand can usually sort out the difficulties that led to the conversion. Hand approaches are preferable for conversions because the final incision length required, in most cases, is likely to be notably shorter than for full open conversions. If for no other reason than conversion after a failed straight laparoscopic case, the minimally invasive surgeon should learn hand-assisted methods.

It has been clearly established that patients benefit from minimally invasive operations in regards to short-term results and limitation of abdominal wall trauma. If our goal is to improve surgical care for our patients then, as educators, we should strive to increase the proportion of colorectal resections that are done nationwide using minimally invasive methods. It is the opinion of a good number of expert minimally invasive surgeons who work at teaching institutions and who are adept at both straight and hand-assisted methods that hand-assisted methods are easier to teach and learn and are associated with a higher adoption rate than straight laparoscopic techniques. It has been the authors experience that it takes 6-12 months to teach a fellow how to take down the splenic flexure independently using straight laparoscopic methods whereas most fellows become proficient at the same task done with hand-assisted methods after 10-15 cases.

Who should not get a hand-assisted operation? Thin and low-BMI patients, in the absence of bulky pathology, are better served with a straight laparoscopic operation. The hand often gets in the way of the camera in these situations and can take up the lion's share of the available working space. These patients are also the ones in whom a $3-5 \mathrm{~cm}$ incision may suffice. Also, although the strong hand proponents would disagree, in the opinion of the authors, moderate-BMI patients and a fair number of higher-BMI patients with nonbulky pathology are best operated via straight laparoscopic means.

We routinely offer minimally invasive resections to all patients, regardless of BMI. Our preference is to use the straight laparoscopic approach. However, we do not hesitate to use hand-assisted methods in situations where it is judged that the final skin incision, after a laparoscopicassisted resection, is likely to be as large as that required for the hand-assisted approach. In addition, hand-assisted methods are also used routinely for low anterior resections for lesions in the distal rectum and in situations where the initial laparoscopic evaluation suggests that the intracorporeal dissection and mobilization will be very difficult.

Over the last 2 years a total of 498 elective colorectal resections were performed at the senior author institution; $87 \%$ were done using minimally invasive methods whereas $13 \%$ were done using open techniques. Of the minimally invasive cases $(n=432), 71 \%$ were done via straight laparoscopic methods whereas in $29 \%$ hand-assisted and hybrid techniques (HAL group) were employed. In a hybrid operation part of the case is done using closed methods after which the resection is completed, the specimen is extracted, and anastomosis constructed via a limited open incision. Hand-assisted/hybrid methods were almost uniformly used for sigmoid and LARs whereas they 
were rarely used for right-sided or proximal left-sided resections.

When the data is examined from the perspective of BMI it is noted that the rate of utilization of HALS/hybrid methods is directly related to the BMI of the patients: $\mathrm{BMI} \leq 33,23 \%$; $\mathrm{BMI}>33,44 \%$; $\mathrm{BMI}>40,50 \%$. The conversion rate also increases with the BMI, straight laparoscopic to a greater extent than hand-assisted methods. The laparoscopic conversion rate ranges from 12\% (BMI $\leq 33)$ to $36 \%$ (BMI > 33), to $67 \%(\mathrm{BMI}>40)$ whereas the hand-assisted/hybrid conversion rate peaks at $29 \%$ (BMI > 40). Finally, the difference in incision length between the two procedures decreases as the BMI rises: $\mathrm{BMI} \leq 33,5.6 \mathrm{~cm}(\mathrm{LAP})$ versus $10 \mathrm{~cm}(\mathrm{HAL})$; $\mathrm{BMI}>33$, $7.7 \mathrm{~cm}$ (LAP) versus $10.7 \mathrm{~cm}(\mathrm{HAL})$; $\mathrm{BMI}>40,11.5 \mathrm{~cm}$ (LAP) versus $11.4 \mathrm{~cm}$ (HAL) [7]. We believe this data supports the selective use of hand-assisted methods and an overall approach that embraces both straight laparoscopic and hand-assisted techniques. Such a philosophy permits maximum utilization of minimally invasive methods which, by virtue of improved short-term results, translates into patient care benefits.

Hand-assisted methods are a tool of the surgeon, as are straight laparoscopic and open methods. The surgeon with the most tools at his disposal will be best equipped to deal with the wide array of pathology and patients (body habitus and BMI) that he is called on to treat. The "best" tool for the task at hand will vary from patient to patient. A surgeon who does either straight laparoscopic or hand-assisted methods exclusively will be handicapped. The selective use of a straight laparoscopic, hand-assisted, or hybrid method permits a minimally invasive approach to be offered to most elective colorectal resection patients regardless of BMI or pathology. Hand-assisted methods decrease the full laparotomy conversion rate and permit the performance of minimally invasive procedures that otherwise would be done using open methods.

\section{References}

1. Mooney MJ, Elliott PL, Galapon DB, James LK, Lilac LJ, O'Reilly MJ (1998) Hand-assisted laparoscopic sigmoidectomy for diverticulitis. Dis Colon Rectum 41(5):630-635

2. Gorey TF, O'riordain MG, Tierney S, Buckley D, Fitzpatrick JM (1996) Laparoscopic-assisted rectopexy using a novel hand-access port. J Laparoendosc Surg 6(5):325-328

3. Nelson H, Sargent DJ, Wieand HS, et al. (2004) A comparison of laparoscopically-assisted and open colectomy for cancer: The Clinical Outcomes of Surgical Therapy Study Group. New Engl J Med 350(20):2050-2059

4. Marcello P, Fleshman JW, Milsom JW, et al. (2007)Hand Assisted vs. Laparoscopic Colorectal Surgery: A Multi-center Prospective Randomized Trial. Presented at 2007 ASCRS Meeting, publication pending in Diseases Colon Rectum

5. Vithiananthan S, Cooper Z, Betten Z, Stapleton G, Carter J, Huang E, Whelan RL (2001) Hybrid laparoscopic flexure takedown and open procedure for rectal resection is associated with significantly shorter length of stay than equivalent open resection. Dis Colon Rectum 44:927-935

6. Tjandra JJ, et al. (2007) Laparoscopic-assisted ultra-low anterior resection: Laparoscopic vs. hand-assisted laparoscopic surgery: a case controlled comparison. Podium presentation at the June 2007 ASCRS meeting in St. Louis, MO

7. Unpublished data from colorectal service at authors' institution. Presbyterian Hospital, Columbia University Health Science Campus, New York, NY 